\title{
Lifetime test on EUV photomask with EBL2
}

Chien-Ching Wu, Markus Bender, Rik Jonckheere, Frank Scholze, Herman Bekman, et al.

Chien-Ching Wu, Markus Bender, Rik Jonckheere, Frank Scholze, Herman

Bekman, Michel van Putten, Rory de Zanger, Rob Ebeling, Jeroen

Westerhout, Kyri Nicolai, Jacqueline van Veldhoven, Véronique de RooijLohmann, Olaf Kievit, Alex Deutz, "Lifetime test on EUV photomask with EBL2," Proc. SPIE 11178, Photomask Japan 2019: XXVI Symposium on Photomask and Next-Generation Lithography Mask Technology, 111780E (27 June 2019); doi: 10.1117/12.2537734

SPIE. Event: Photomask Japan 2019, 2019, Yokohama, Japan 


\title{
Lifetime test on EUV photomask with EBL2
}

\author{
Chien-Ching $\mathrm{Wu}^{1}$, Markus Bender ${ }^{2}$, Rik Jonckheere ${ }^{3}$, Frank Scholze ${ }^{4}$, Herman Bekman ${ }^{1}$, \\ Michel van Putten ${ }^{1}$, Rory de Zanger ${ }^{1}$, Rob Ebeling ${ }^{1}$, Jeroen Westerhout ${ }^{1}$, Kyri Nicolai ${ }^{1}$, \\ Jacqueline van Veldhoven ${ }^{1}$, Véronique de Rooij-Lohmann ${ }^{1}$, Olaf Kievit ${ }^{1}$, Alex Deutz ${ }^{1}$ \\ ${ }^{1}$ TNO, Stieltjesweg 1, 2628 CK Delft (Netherlands) \\ ${ }^{2}$ Advanced Mask Technology Center GmbH \& Co KG, Rähnitzer Allee 9, 01109 \\ Dresden(Germany) \\ 3 imec, Kapeldreef 75, 3001 Leuven (Belgium) \\ ${ }^{4}$ Physikalisch-Technische Bundesanstalt, 38116 Braunschweig, (Germany)
}

\begin{abstract}
TNO has built EBL2, an EUV exposure facility equipped with an in vacuo X-ray photoelectron spectroscopy setup (XPS) and an in-situ ellipsometer. EBL2 enables lifetime testing of EUV optics, photomasks, pellicles and related components under development in relevant EUV scanner and source conditions, which was previously not available to industry. This lifetime testing can help the industry to prepare for high volume production using EUV lithography by bringing forward information about material behavior which facilitates the development cycle.

This paper describes an EUV photomask lifetime test performed at EBL2. The mask was exposed to different EUV doses under a controlled gas and temperature environment. To investigate how EUV light interacts with the mask, various analysis techniques were applied before and after EUV exposure. In-situ XPS was used to investigate elemental compositions of the mask surface. An ex-situ critical dimension scanning electron microscope (CD-SEM) and an atomic force microscope (AFM) were used to explore the impact of EUV light on critical dimensions (CD) and feature profiles. In addition, EUV reflectometry (EUVR) was used to investigate the change of reflectivity after EUV exposures. The exposure conditions are reported, as well as an analysis of the effects observed.
\end{abstract}

Keywords: EBL2, EUV lithography, X-ray photoelectron spectroscopy (XPS), photomask, lifetime

\section{INTRODUCTION}

As EUV scanners become increasingly mature products, EUV lithography is expected for high volume manufacturing (HVM) in 2019. In order to have higher throughput, the ASML EUV source power roadmap aims for $500 \mathrm{~W}$ and even beyond this. The increase of source power indicates that components, including optics, photomasks and pellicles used in EUV scanners have to withstand higher EUV intensities and avoid degradation during their guaranteed life time. Therefore, there is a growing demand to investigate the effects of such high EUV powers on these components in scanner relevant environments. For this purpose, TNO has built an EUV exposure facility named EUV beam-line two (EBL2) equipped with in-situ imaging ellipsometer and in-vacuo XPS. A more detailed description can be found in previous publications $[1],[2],[3]$.

It is known that the current EUV photomask with the required 50-70nm thickness of the Ta-based metallic absorber layer does not offer optimal wafer images. Alternative metal absorbers with higher absorptivity than $\mathrm{Ta}$, such as $\mathrm{Ni}$ and Co have been proposed and, in simulation, show improved imaging at $<40 \mathrm{~nm}$ thickness ${ }^{[4]}$. These new absorber materials must not only meet the criteria for improved imaging, but also be compatible with all process steps and be able to withstand the scanner environment and EUV illumination conditions. It has been reported that an EUV photomask showed bulge-like damages after exposing approximately 40000 wafers ${ }^{[5]}$. This result suggests that EUV dose may play a role for the lifetime of photomasks. Apart from lifetime studies, investigating the impact of contamination on photomasks is also important for

Photomask Japan 2019: XXVI Symposium on Photomask and Next-Generation Lithography Mask Technology, edited by Akihiko Ando, Proc. of SPIE Vol. 11178, 111780E (c) The Authors. Published under a Creative Commons Attribution CC BY 3.0 License · doi: 10.1117/12.2537734 
an HVM foundry fab. Molecular contamination can build up on an EUV photomask during storage. Upon subsequent EUV exposure these loosely bound hydrocarbons can be carbonized, leading to EUV photomask reflection loss and degradation of exposure uniformity ${ }^{[6]}$.

EBL2 is intended to study (accelerated) photomask lifetime and the impact of EUV-induced contamination. For this purpose, EBL2 facilitates automated handling of a full size EUV photomask ${ }^{[7]}$. This paper describes a lifetime test on an EUV photomask by using EBL2. This photomask was exposed to EUV light with varied doses in a gas environment representative for an ASML NXE:3400 EUV scanner. Subsequently the photomask was analyzed by in-situ XPS, and exsitu by SEM, AFM and EUVR. The exposure conditions as well as an analysis of the effects observed are reported here.

\section{EXPERIMENTAL}

A test EUV photomask supplied by Advanced Mask Technology Center GmbH \& Co KG (AMTC) was used for a lifetime test at EBL2. This photomask contains a regular grid of 144 Test-clips named QA cells in 4 different densities of fill patterns: $0 \%, 25 \%, 50 \%$ and $75 \%$. The $0 \%$ indicates that the top layer of the photomask is only absorber without any special fill patterns. The QA cells consist of different patterns in terms of size, feature types and tones (lines or spaces). Figure 1 shows the patterns overview on the test EUV photomask.

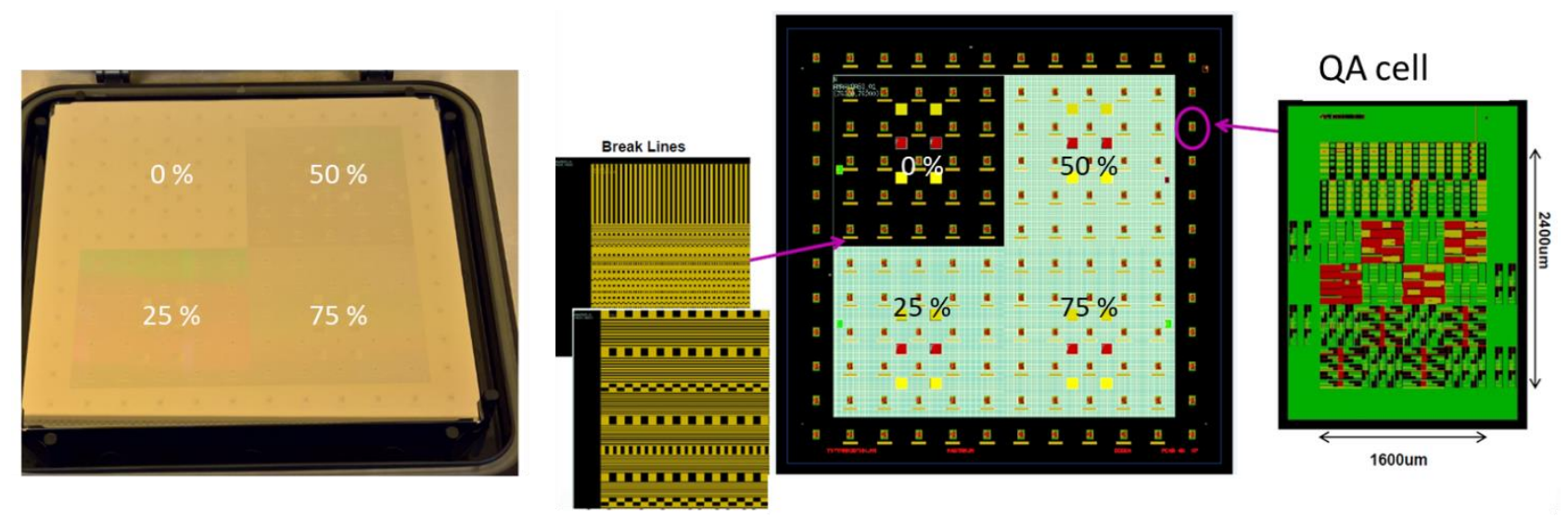

Figure 1 Overview of patterns on the test EUV photomask.

The experimental procedure is illustrated in Figure 2(a). Before the photomask was loaded into EBL2 for EUV exposure, it was pre-characterized by CD-SEM and AFM at AMTC, and by XPS at TNO. To verify the EUV dose impact on a mask, five QA cells (Pos. 1 to Pos. 5) were selected for EUV exposures, as shown in Figure 2(b). The varied doses applied on the photomask are summarized in Table 1. Positions 1 to 3 received a smaller EUV dose, roughly equal to one thousand wafers exposure on the EUV scanner. Position 5 received the largest EUV dose, roughly equal to ten thousand wafers exposure. The dose calculation is based on the source power at intermediate focus equal to $250 \mathrm{~W}$. The Ref. position shown in Figure 2(b) refers to no direct EUV light on this QA cell.

The photomask was illuminated with a de-focused EUV spot. The broad band EUV light was operated at a repetition rate of $2.7 \mathrm{kHz}$ and its peak intensity for all positions was about $17-18 \mathrm{~mW} / \mathrm{mm}^{2}$. During EUV exposure, the photomask was placed against the sample chuck. The temperature of the chuck was set to $-20^{\circ} \mathrm{C}$ to avoid overheating the photomask. 
(a)

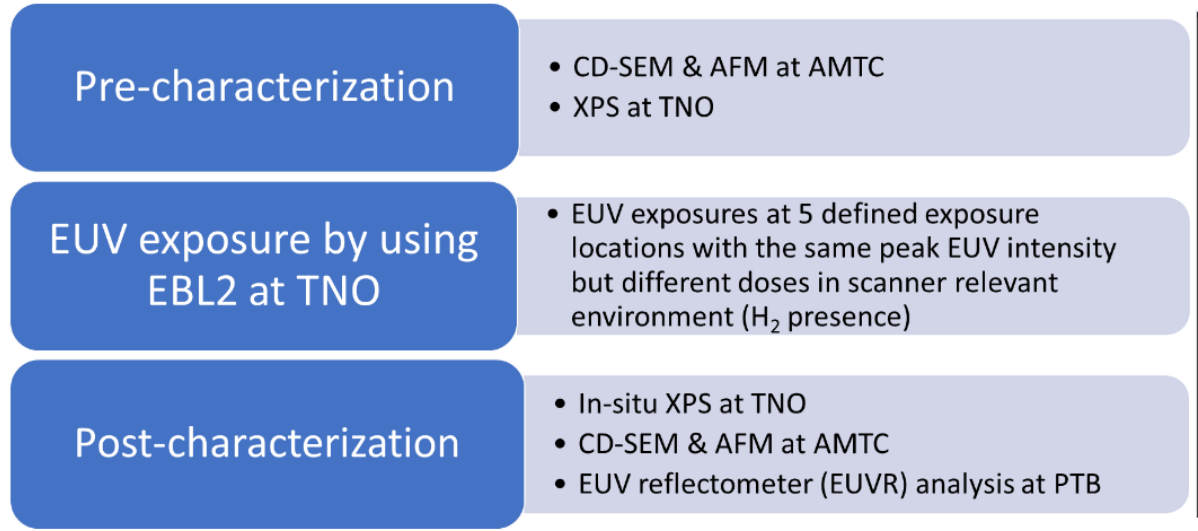

(b)

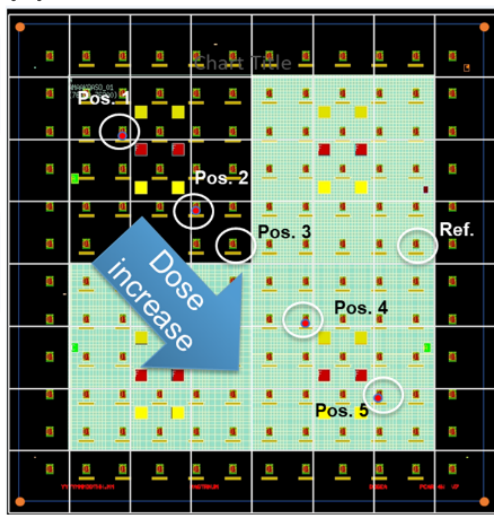

Figure 2 (a) Flowchart illustrating the experimental procedures of lifetime test on the photomask. (b) Illustration of defined QA cells on the test photomask for EUV exposure at EBL2.

Table 1 Summary of the doses applied to the exposed QA cells.

\begin{tabular}{|crc|} 
Positions & $\begin{array}{r}\text { Estimated eq. wafers } \\
\text { exposure }(\#)\end{array}$ & $\begin{array}{c}\text { Peak dose } \\
\left(\mathbf{k J} / \mathrm{cm}^{2}\right)\end{array}$ \\
\hline Pos. $1-3$ & 1.000 & 9 \\
\hline Pos. 4 & 5.000 & 43 \\
\hline Pos. 5 & 10.000 & 93 \\
\hline
\end{tabular}

The 2D EUV intensity map at the defocus position was measured by a calibrated diode before the actual lifetime test as shown in Figure 3 (a). The EUV source was operated at a repetition rate of $200 \mathrm{~Hz}$ during the intensity measurement. The full width at half maximum (FWHM) of the central EUV spot in X and Y-direction is about $2 \mathrm{~mm}$ and $3 \mathrm{~mm}$, respectively. This means the central EUV spot can cover one QA cell. The outer ring of the EUV spot can affect the area outside the defined QA cell. The QA cell was visible by ellipsometer camera as shown in Figure 3(b). In this case, the EUV exposure can be performed at the defined QA cells precisely.

(a)

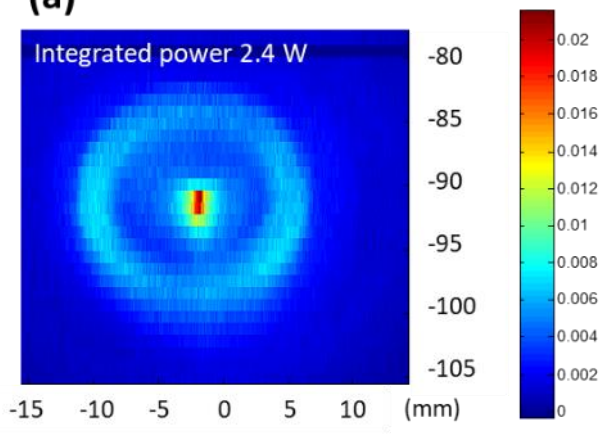

(b)

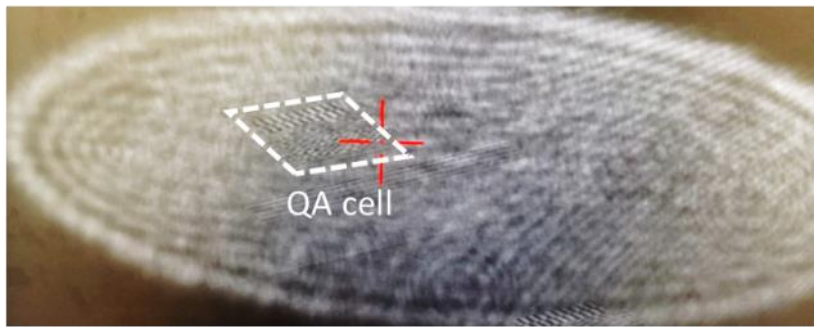

Figure 3 (a) 2D EUV intensity map at defocus position. (b) Photo with a visible QA cell taken by ellipsometer camera. 


\section{RESULTS}

\subsection{XPS}

After EUV exposure the photomask was transferred to the XPS without breaking vacuum. The optical microscope attached to the XPS enables finding the exposed QA cells easily for post-characterization. The XPS results, including atomic percentages (at $\%$ ) of detected elements and oxidation degrees (\%) of key elements, are summarized in Table 2. The oxidation degrees were calculated by splitting peaks of the obtained XPS spectra. Table 2 shows that both carbon and oxygen amounts decrease after EUV exposure. Silicon $(\mathrm{Si})$, tantalum $(\mathrm{Ta})$ and ruthenium $(\mathrm{Ru})$ were partially oxidized before EUV exposure. The oxidation degrees of Si and Ta remain similar after EUV exposure. For Ru, its oxidation degree dramatically reduced after EUV exposure. It is interesting to notice that the Ru oxidation degree of Ref. location shows similar chemical reduction of ruthenium-oxide. This result implies that EUV induced plasma may have impact on a large area. The XPS results of the measured locations treated with different doses show similar results.

Table 2 XPS analysis report pre and post EUV exposure at various positions on the photomask.

\begin{tabular}{|c|c|c|c|c|c|c|c|c|c|c|c|c|c|c|c|c|c|c|}
\hline \multirow{2}{*}{ Description } & \multirow{2}{*}{ Locations } & \multirow{2}{*}{$\begin{array}{l}\text { Peak dose } \\
\left(\mathrm{k} / \mathrm{cm}^{2}\right)\end{array}$} & \multicolumn{7}{|c|}{ at\% (selective key elements normalized to $100 \%$ without C) } & \multirow[b]{2}{*}{ Ta $4 f$} & \multirow[b]{2}{*}{$\mathrm{C}_{1}^{*}$} & \multicolumn{4}{|c|}{ Peak splitting (oxidaiont degree \%) } & \multirow[b]{2}{*}{ Ru non-0x } & \multirow[b]{2}{*}{ Ru-OXA } & \multirow[b]{2}{*}{ Ru-OxB } \\
\hline & & & $F$ is & Mo 3d5/2 & $\mathrm{N}$ 1s & $01 \mathrm{~s}$ & Ru 3d & S2p & Si 2p & & & Si-non $0 x$ & Si-Ox & Ta-non $0 x$ & Ta- $0 x$ & & & \\
\hline \multirow{4}{*}{ Before EUV exposure } & QA cell above Pos 3 & - & 1.1 & 0.6 & 2.0 & 63.2 & 10.1 & $<$ & 7.2 & 15.7 & 23.6 & 51 & 49 & 16 & 84 & 48 & 43 & 10 \\
\hline & QA cell above Pos 4 & - & 1.4 & 0.6 & 1.5 & 63.1 & 12.1 & $<$ & 7.8 & 13.6 & 26.8 & 51 & 49 & 15 & 85 & 48 & 45 & 7 \\
\hline & QA cell above Pos 5 & - & 1.7 & 0.6 & 1.4 & 63.3 & 12.0 & $<$ & 7.6 & 13.5 & 25.8 & 53 & 47 & 15 & 85 & 48 & 44 & 8 \\
\hline & Ref. & - & 1.4 & 0.6 & 1.5 & 63.3 & 11.4 & $<$ & 7.5 & 14.2 & 25.7 & 51 & 49 & 14 & 86 & 47 & 44 & 9 \\
\hline \multirow{4}{*}{$\begin{array}{c}\text { After EUV exposure (in } \\
\text { vacuo XPS analysis) }\end{array}$} & Pos 3 & 9 & 0.3 & 1.2 & 1.1 & 53.4 & 16.9 & 0.4 & 11.6 & 15.1 & 8.5 & 52 & 48 & 17 & 83 & 98 & 2 & 0 \\
\hline & Pos 4 & 43 & 0.1 & 1.0 & 0.9 & 54.6 & 15.6 & 0.4 & 11.7 & 15.6 & 7.0 & 50 & 50 & 15 & 85 & 98 & 2 & 0 \\
\hline & Pos 5 & 93 & 0.4 & 1.0 & 0.7 & 53.9 & 16.7 & 0.5 & 11.6 & 15.2 & 6.7 & 50 & 50 & 16 & 84 & 98 & 2 & 0 \\
\hline & Ref. & - & 0.5 & 1.2 & 1.0 & 52.6 & 16.7 & 0.3 & 11.8 & 15.9 & 8.4 & 54 & 46 & 17 & 83 & 97 & 3 & 0 \\
\hline
\end{tabular}

\subsection{CD-SEM \& AFM}

The difference of CD was measured by CD SEM for each isolated clear (IC), isolated dark (ID), dense clear (DC), dense dark (DD) features with target sizes of $180 \mathrm{~nm}, 120 \mathrm{~nm}$, and $80 \mathrm{~nm}$. The results of CD difference before and after exposure for different target sizes are similar. Therefore, only the CD difference result of $180 \mathrm{~nm}$ feature size is shown in Figure 4(a). Point-to-point CD differences between exposure and non-exposure areas are similar.

The AFM results of absorber heights are shown in Figure 4(b). It seems the absorber height increases after exposure. The increase in height is within the AFM measurement uncertainty $(\sim 0.7 \mathrm{~nm})$, and therefore it is difficult to conclude if the absorber height changes due to EUV treatment.

(a)

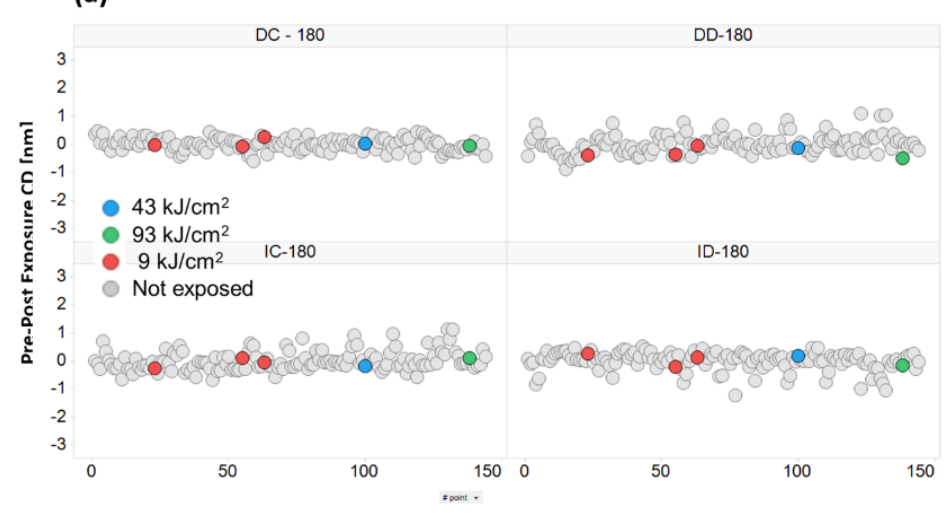

(b)

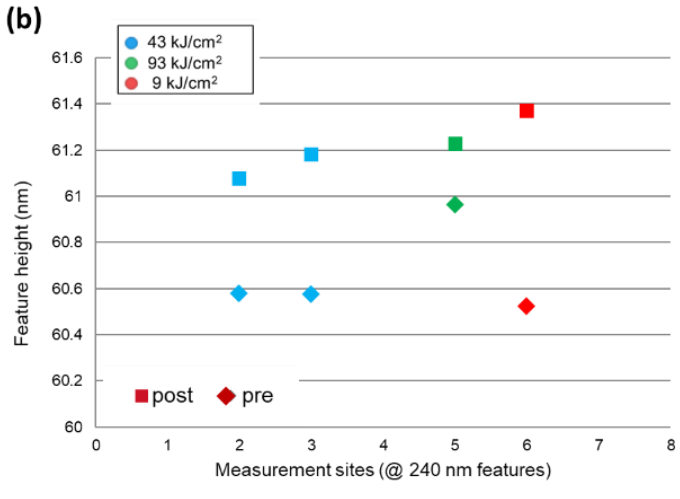

Figure 4 (a) Results of CD difference of each isolated clear (IC), isolated dark (ID), dense clear (DC), dense dark (DD) features with target sizes of $180 \mathrm{~nm}$. (b) Absorber heights measured by AFM before and after EUV exposure. 


\subsection{EUV reflectivity (EUVR)}

EUVR of the exposed mask was measured by PTB's reflectometer at the EUV beamline at the MLS storage ring. A full EUVR map with a step size of $2 \mathrm{~mm}$ and local detailed EUVR maps with a step size of $1 \mathrm{~mm}$ were performed. The photon beam sizes for the full map and local detailed maps are $2 \mathrm{~mm} \times 2 \mathrm{~mm}$ and $1 \mathrm{~mm} \times 1 \mathrm{~mm}$, respectively. The local detailed EUVR maps of Pos. 3 to Pos. 5 are shown in Figure 5. Reflectivity loss rings surrounding the QA cell subjected to higher doses (Pos. 4 and Pos. 5) are observed. These EUVR loss rings correspond to the outer ring of the EUV spot (see Figure 5) and also confirm that the EUV light exposed to the targeted locations well. There was no EUVR pre-characterization done on this mask. The local variation of the reflectance of the bright fill pattern in the fourth quadrant (exposure Pos. 4 $\&$ Pos. 5), however, strongly suggests that there is a correlation of reflectance loss and exposure dose in bright areas. Note that the other spots with lower dose in the dark quadrant did not show any detectable footprint. From this, however, no clear conclusion can be drawn without a detailed pre-characterization. Therefore, it should not be concluded from the results shown that low EUV dose does not result in EUVR loss.
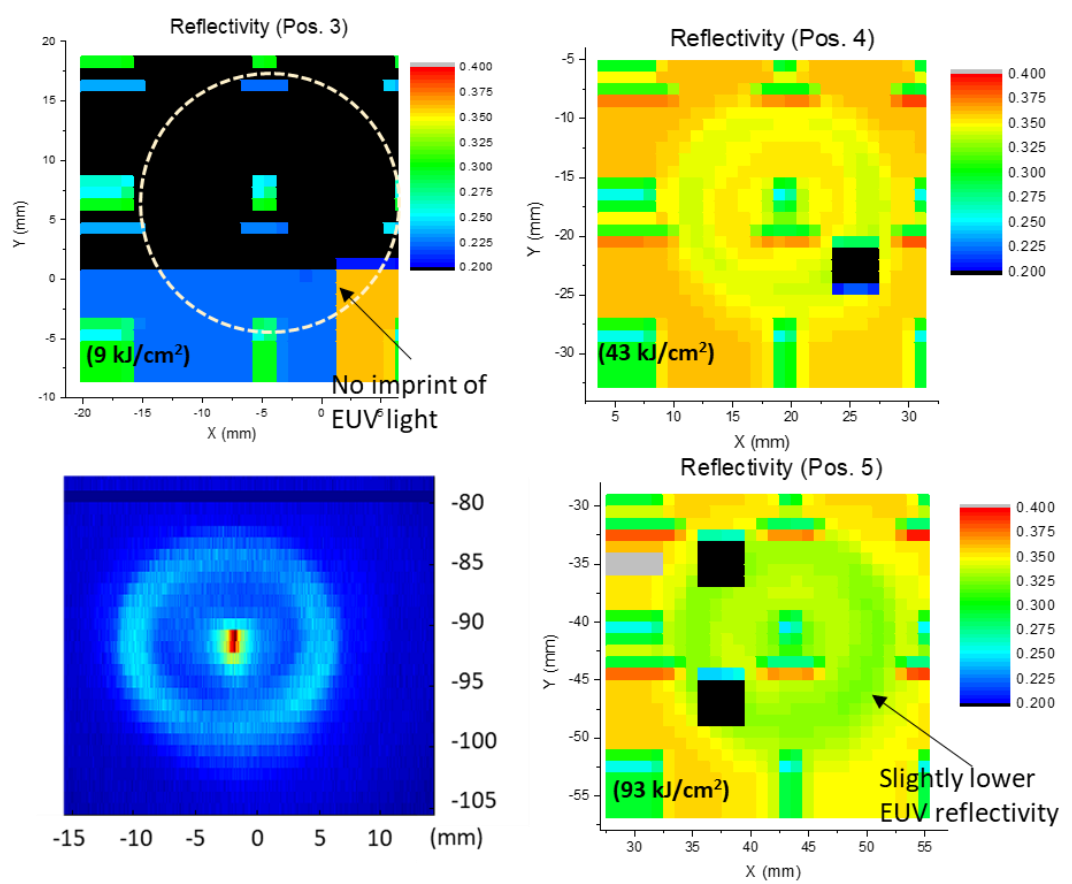

Figure 5 Local detailed EUVR map of Pos. 3 to Pos. 5 and the EUV intensity map of EBL2 at the conditions used in the exposure. The color scale of each EUVR map is the same. The outer ring of the EUV intensity map matches well with the reflectivity loss ring. The EUVR of the exposed absorber is shown to decrease compared to the background.

\section{SUMMARY \& OUTLOOK}

A lifetime test on an EUV photomask in a scanner-relevant environment using EBL2 is reported. Different analysis techniques were used to assess the mask degradation over lifetime. The surface results of this EUV exposed photomask are summarized in Table 3. In this lifetime test, a photomask with non-uniform patterns are used. Therefore, it is difficult to conclude if the observed effects result from the $\mathrm{Ru}$ on the multilayer area or from the patterned absorber. To further investigate the cause of the observed effects, similar EUV lifetime tests will be performed on a mask with a clear Ru-onmultilayer area and on a mask with patterned absorber with known pattern density, separately. Eventually, a full spec EUV photomask exposed at EBL2 will be tested in an NXE scanner in order to assess the impact of the EBL2 exposure on wafer printing. 
Table 3 Summary of analysis results of the EUV exposed photomask.

\begin{tabular}{|c|c|c|c|}
\hline $\begin{array}{l}\text { Characterization } \\
\text { techniques }\end{array}$ & $\begin{array}{l}\text { Before EUV } \\
\text { exposure }\end{array}$ & $\begin{array}{l}\text { After EUV } \\
\text { exposure }\end{array}$ & Key Results \\
\hline XPS & $\sqrt{ }$ & $\sqrt{ }$ (in situ) & $\begin{array}{l}\text { - Ruthenium-oxide is reduced after EUV exposure. } \\
\text { - Carbon and oxygen amounts decrease after EUV exposure. } \\
\text { - No obvious difference among positions exposed with various } \\
\text { EUV doses. } \\
\text { - EUV induced plasma may have impact on large area. }\end{array}$ \\
\hline CD-SEM & $\sqrt{ }$ & $\sqrt{ }$ & $\begin{array}{l}\text { Point-to-point CD difference before and after EUV exposure } \\
\text { shows no obvious changes. }\end{array}$ \\
\hline AFM & $\sqrt{ }$ & $\sqrt{ }$ & $\begin{array}{l}\text { - Absorber height after EUV exposures increases but within AFM } \\
\text { height measurement uncertainty }(\sim 0.7 \mathrm{~nm}) \text {. }\end{array}$ \\
\hline EUVR & n.a. & $\sqrt{ }$ & $\begin{array}{l}\text { - Shape of EUV light is visible on the EUVR map. } \\
\text { - Position exposed to the highest EUV dose shows slightly lower } \\
\text { EUV reflectivity. }\end{array}$ \\
\hline
\end{tabular}

\section{ACKNOWLEDGEMENTS}

This project has received funding from the Electronic Component Systems for European Leadership Joint Undertaking under grant agreement No 737479. This Joint Undertaking receives support from the European Union's Horizon 2020 research and innovation programme and Netherlands, Italy, Germany, Belgium, France, Czech Republic, Hungary, Israel. In addition, TNO received funding via the Dutch ministry of Economic Affairs (NanoLabNL and Toekomstfonds).

\section{REFERENCES}

[1] te Sligte, E., Koster, N., Molkenboer, F., Deutz, A., "EBL2, a flexible, controlled EUV exposure and surface analysis facility," Proc. of SPIE 9984, 99840R (2016).

[2] te Sligte, E., Koster, N., Molkenboer, F., van der Walle, P., Muilwijk, P., Mulckhuyse, W., Oostdijck, B., Hollemans, C., Nijland, B., Kerkhof, P., van Putten, M., Hoogstrate, A., Deutz, A., "EBL2: high power EUV exposure facility," Proc. of SPIE 9985, 998520-1 (2016).

[3] Koster, N., te Sligte, E.; Molkenboer, F., Deutz, A., van der Walle, P., Muilwijk, P., Mulckhuyse, W., Oostdijck, B., Christiaan Hollemans, C., Nijland, B., Kerkhof, P., van Putten, M., Westerhout, J., "First light at EBL2," Proc. of SPIE 10143, 101431N (2017).

[4] Philipsen, V., Luong; K.M., Souriau; L., Altamirano-Sánchez, E., Adelmann, C., Laubis, C., Scholtze, F., Kruemberg, J., Reuter, C., Hendrickx, E., "Single element and metal alloy novel EUV mask absorbers for improved imaging," Proc. of SPIE 10450, 104500G (2017).

[5] Kim, S.-S., Chalykh, R., Kim, H., Lee, S., Park, C., Hwang, M., Park, J.-O., Park, J., Kim, H., Jeon, J., Kim, I., Lee, D., Na, J., Kim, J., Lee, S., Kim, H., Nam, S.-W., "Progress in EUV lithography toward manufacturing" Proc. of SPIE 10143, 1014306 (2017)

[6] Singh, S., Yatzor, B., Taylor, R., Wood, O., Mangat, P. "A study on EUV photomask surface molecular contamination under different storage conditions in a HVM foundry fab," Proc. of SPIE 10143, 101431T (2017).

[7] Wu, C.-C., Sligte, E., Bekman, H., Storm, A., van Putten, M., Limpens, M., van Veldhoven, J., Deutz, A., "EUV mask lifetime testing using EBL2," Proc. of SPIE 10583, 1058310 (2018). 